\title{
A quantitative analysis of nozzle surface-bound fuel for diesel injectors
}

\author{
Jack Turner ${ }^{1}$, Dan Sykes ${ }^{1}$, Guillaume de Sercey ${ }^{1}$, Viacheslav Stetsyuk ${ }^{1}$, Martin Gold², \\ Richard Pearson ${ }^{2}$, Cyril Crua ${ }^{1}$ \\ ${ }^{1}$ Advanced Engineering Centre, University of Brighton, Brighton, UK \\ ${ }^{2}$ BP Formulated Products Technology, Pangbourne, UK \\ *Corresponding author: j.turner3@brighton.ac.uk
}

\begin{abstract}
In a fuel injector at the end of the injection, the needle descent and the rapid pressure drop in the nozzle leads to discharge of large, slow-moving liquid structures. This unwanted discharge is often referred as fuel 'dribble' and results in near-nozzle surface wetting, creating fuel-rich regions that are believed to contribute to unburnt hydrocarbon emissions. Subsequent fluid overspill occurs during the pressure drop in the expansion stroke when residual fluid inside the nozzle is displaced by the expansion of trapped gases as the pressure through the orifices is equalised, leading to further surface wetting. There have been several recent advancements in the characterisation of these near nozzle fluid processes, yet there is a lack of quantitative data relating the operating conditions and hardware parameters to the quantity of overspill and surface-bound fuel. In this study, methods for quantifying nozzle tip wetting after the end of injection were developed, to gain a better understanding of the underlying processes and to study the influence of engine operating conditions. A high-speed camera with a longdistance microscope was used to visualise fluid behaviour at the microscopic scale during, and after, the end of injection. In order to measure the nozzle tip temperature, a production injector was used which was instrumented with a type $\mathrm{K}$ thermocouple near one of the orifices. Image post-processing techniques were developed to track both the initial fuel coverage area on the nozzle surface, as well as the temporal evolution and spreading rate of surface-bound fluid. The conclusion presents an analysis of the area of fuel coverage and the rate of spreading and how these depend on injection pressure, in-cylinder pressure and in-cylinder temperature. It was observed that for this VCO injector, the rate of spreading correlates with the initial area of fuel coverage measured after the end of injection, suggesting that the main mechanism for nozzle wetting is through the impingement of dribble onto the nozzle. However, occasional observations of the expansion of orifice-trapped gas were made that lead to a significant increase in nozzle wetting.
\end{abstract}

\section{Keywords}

Diesel; end of injection; dribble; surface wetting; image processing

\section{Introduction}

Increasingly strict emissions standards place considerable pressure on the automotive industry to increase the efficiency of, and reduce the emissions of the engine. This is primarily done by optimising the fuel-air mixing processes that occur inside the combustion chamber. Since the fuel air mixing is affected by the fuel injection equipment (FIE), much of the research efforts have been in improving the injector design and spray characteristics. This has resulted in the numbers of holes in the injector tip increasing with subsequent designs, as well as increases in the operating pressure, with typical systems operating with common rail pressures of over $200 \mathrm{MPa}$. These increasingly harsh conditions are believed to accelerate the formation and growth of deposits in and around the injector tip.

The accumulation of deposits in and around injector tips is associated with reduced engine performance and lifetime. This reduction in performance is known to manifest in a variety of ways including increased acoustic and pollutant emissions [1-3]. The deposits can also reduce the hydraulic diameter of the nozzle hole, resulting in a reduction in the quantity of injected fuel and reduced quality and consistency of injection [3-5]; all of which cause a reduction in engine power [6]. The deposits can also increase cavitation, which can then lead to further coking of the nozzle [7]. Injector needle sticking can occur and eventually injector failure [8, 9]. The issue of deposit formation is further compounded by both the strict tolerances of critical parts of the FIE 
Recent efforts have been directed at optimisation of the injection event itself. Shifts towards piezo-actuated injectors mean that dynamic response times are improved, reducing the time spent in the transient injection phase. This has been shown to give superior fuel air mixing when compared with older systems using solenoid actuated injectors $[5,6]$. A more recent technique in injection optimisation utilises split injection strategies. This gives increased control of the rate of injection. Split injections have been shown to reduce unburnt hydrocarbon (UHC) emissions. There are, however, diminishing returns, suggestive of a persistent source of UHC emissions [10].

Transient injection phases, in particular the end of injection, are believed to be related to this persistent source of emissions [11], as well as the continuing issues around injector deposits. During the end of injection, as the needle descends, the complex fluid flow inside the nozzle and orifices results in the discharge of large, slow and deformed liquid structures during the collapse of the spray. This leads to two problems. Firstly, the liquid often lands on the near-nozzle surface where it is then exposed to the high pressures and temperatures inside the cylinder. Since this creates locally rich regions in an overall lean environment, incomplete combustion, cracking and degradation are likely to occur. This is particularly true if the combustion flame front contacts the surface-bound fluid. It is likely that this process would impact the initial formation of carbonaceous deposits in and around the injector tip. Secondly, the large, slow-moving droplets that were ejected may not be atomized sufficiently to undergo complete evaporation, thus producing unwanted combustion by-products. There is also potential for these droplets to be drawn directly through the exhaust valve where they contribute directly to the UHC emissions. Increased use of split injection strategies increases the time spent by the spray within the transient regime. This increases the occurrence of liquid structures associated with the end of injection and the related problems with emission and deposits. As such, the end of injection has recently attracted considerable efforts to characterise and explain the observed phenomena [12-15].

There have been numerous simulations and numerical studies that have predicted the complex fluid behaviour inside nozzle during the closing of the needle [16-19] these studies predict the breaking up of the jet into ligaments as well as rapid pressure fluctuations during this time. There is a point in which the experimental observation and numerical simulations are in very good agreement. There does, however, exist a lack of quantitative analysis relating to the volume of fluid on the surface of the near nozzle region after the end of injection. There have been simulations on predicting mass outflux [18], but this includes fluid that is discharged into the cylinder as well as onto the surface.

Whilst there are interesting fluid dynamic processes occurring at the orifices throughout the engine cycle [15, 20], this investigation is focused on nozzle wetting processes that occur within a few milliseconds from the end of injection. Development of quantitative methods to measure both the spreading area of surface-bound fluid in the near-nozzle region after the end of injection, as well as the spreading rate, allows elucidation of the underlying mechanism of the initial fuel spreading. In order to decouple the effects of pressure and momentum on the initial coverage area, injections were carried out at significantly different timing during the engine cycle, resulting in greatly different in-cylinder pressure and temperature. This allowed the effect of capillary action and spreading to be investigated since the physical properties of the fuel are functions of pressure and temperature and the spreading dynamics are a function of both liquid physical properties and ambient conditions. In fact, at higher pressures and temperatures nozzle surface wetting is expected to increase. This is due to the pressure effect on viscosity and the temperature effect on viscosity and surface tension. Increasing both pressure and temperature relaxes the contact angle and increases surface wetting [21]. Based on this it can be hypothesised that the surface wetting will be more pronounced when injecting at top-dead-centre (TDC). Given the timescales involved, it is likely that pressure would be the dominant effect, since the nozzle tip will experience a reduced temperature variation due to thermal inertia.

\section{Experimental setup}

The principal apparatus used in this research was a single cylinder reciprocating rapid compression machine. The fuel was delivered by a Delphi common-rail system, comprising a DFP-3 high-pressure pump rated at $200 \mathrm{MPa}$, and a six-hole Siemens instrumented injector with a VCO type nozzle. The setup allows observation of all six orifices simultaneously as they all sit within the plane of focus. The injector contained two integrated type $\mathrm{K}$ thermocouples accurate to $\pm 1 \mathrm{~K}$. These thermocouples record temperature at a point just below the tip of the nozzle surface and another inside the body of the injector to measure the fuel temperature around the needle prior to discharge. The injector was mounted orthogonal to the incident light to allow visualisation of all orifices simultaneously. 
Table 1. Test conditions and hardware characteristics

\begin{tabular}{c|c}
\hline \hline Parameter & Value \\
\hline Nozzle type & Siemens DW10, 6-hole VCO \\
Orifice diameter & $145 \mu \mathrm{m}$ \\
Peak motored pressure & $50 \mathrm{bar}$ \\
Peak motored temperature & $640 \mathrm{~K}$ \\
Injection pressures & $500 ; 1500 \mathrm{bar}$ \\
Injection timing & $-30 ; 0 ; 30 \mathrm{CA}$ aTDC \\
Injection pulse duration & $200 ; 400 ; 600 \mu \mathrm{s}$ \\
Nozzle tip temperature & $405 \mathrm{~K}+/-3 \mathrm{~K}$
\end{tabular}

The light source consisted of two synchronised high-speed lasers illuminating the opposite sides of a 6-hole Siemens DW10 instrumented injector. Laser 1 is a CAVITAR Cavilux solid state diode laser emitting at $690 \mathrm{~nm}$ and Laser 2 is a copper vapour laser emitting at 510 and $578.2 \mathrm{~nm}$ with the beam being delivered via optical fibre. Laser 1 is controlled via a software interface and can be used to trigger a signal generator which, in turn, emits a pulse to drive Laser 2. The frame exposure of the high-speed camera was set at $1 \mu \mathrm{s}$, although the exposure was controlled by the shorter laser pulse durations. More details can be found in the specification table below.

Table 2. Imaging equipment specification. Frame rates shown are at minimum and maximum resolution and then a representative resolution from the data presented in this project.

\begin{tabular}{c|c}
\hline \hline Parameter & Value \\
\hline \hline Camera & Phantom V12.1 \\
Pixel size & $20 \mu \mathrm{m}$ \\
Bit depth & $12-\mathrm{bit}$ \\
Frame exposure time & $1 \mu \mathrm{s}$ \\
Frame rate $(704 \times 704$ pixels $)$ & $10,000 \mathrm{fps}$ \\
Image resolution & $6.58 \mu \mathrm{m}$ per pixel \\
Field of view & $4.63 \mathrm{~mm} \times 4.63 \mathrm{~mm}$
\end{tabular}

With this setup, video data was acquired showing all six orifices for one entire engine revolution. The orifice were numbered clockwise as seen in Figure 1.

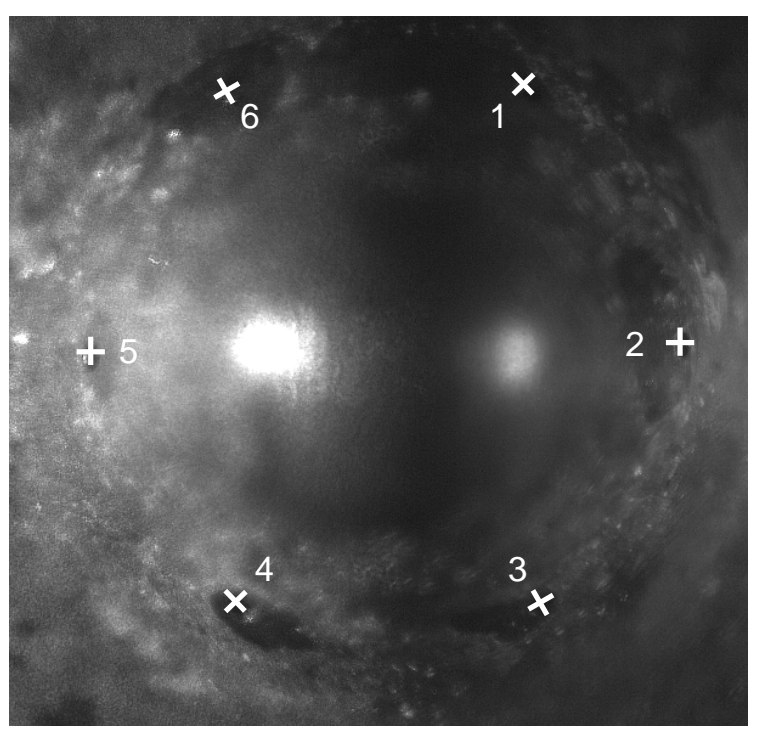

Figure 1. Video frame showing the entire injector tip and all orifices in focus. Note how each orifice is illuminated differently, in particularly how the equatorial orifices 2 and 5 seem to afford little contrast as they lie directly in the path of the incident laser light. The remaining orifices 1,3,4,6 offer better contrast as light from the liquid layers is scattered away from the imaging system. The surface-bound fluid appears darker, whereas this effect is reduced for orifices 2 and 5 . 


\section{Quantification of coverage area and spreading rate}

In order to obtain quantitative data to explore the key hypothesis that the surface wetting will be more pronounced when injecting at TDC, image processing algorithms were developed to semi-automatically extract the coverage area of liquid fuel on the nozzle tip. The key stages of the image processing algorithm are listed and described below:

1. Normalisation of pixel intensities: the intensity histogram was stretched in relation to the top and bottom $10 \%$ pixel intensities across the frame to account for laser pulse to pulse variation.

2. Flat fielding: the illumination across the frame was not homogeneous due to the curvature of the nozzle tip surface. The intensities across the frame were homogenised using a non-linear gradient filter.

3. Removal of high frequency noise: high frequency noise was removed, and small regions with similar pixel intensity distributions were merged using morphological closing with a non-flat, inverted ball-shaped structuring element, followed by morphological opening.

4. Removal of reflections from the orifice edge: an elliptical mask layered over the orifice with constant pixel intensities, equal to the mean of those surrounding it, was applied to remove high intensity reflections from the orifice's edge.

5. Semi-automatic binarisation: a function using the mean and spread of pixel intensities across the frame, in conjunction with a user input coefficient, was used for the binarisation threshold. The automatic thresholding performed well, but a user adjustment was required in $33 \%$ of the videos to faithfully track the coverage area. These tended to be for higher injection pressure conditions.

6. Smoothing of the binarised image: morphological operators were applied to remove small-scale features.

Binarised regions that did not overlap with the orifice were removed, therefore videos showing no coverage did not show any binarised regions. The fuel coverage area was converted from pixels to $\mathrm{mm}^{2}$ via scaling of the final binarised region, accounting for the $30^{\circ}$ inclination of the nozzle tip surface with respect to the optical axis. A visual inspection of the image processing output was performed for all frames to ensure that the automated measurements accurately represented the coverage area. Videos which could not be processed were discarded, and some videos were re-processed with a custom binarisation threshold.

This approach gave consistent results for orifice 3 due to its orientation relative to the illumination and the imaging system, therefore the image processing was only applied in the area surrounding orifice 3 . Visual inspection of the videos confirmed that this particular orifice was representative of the nozzle's behaviour. An example of the user interface for the image processing code is shown in Figure 2.
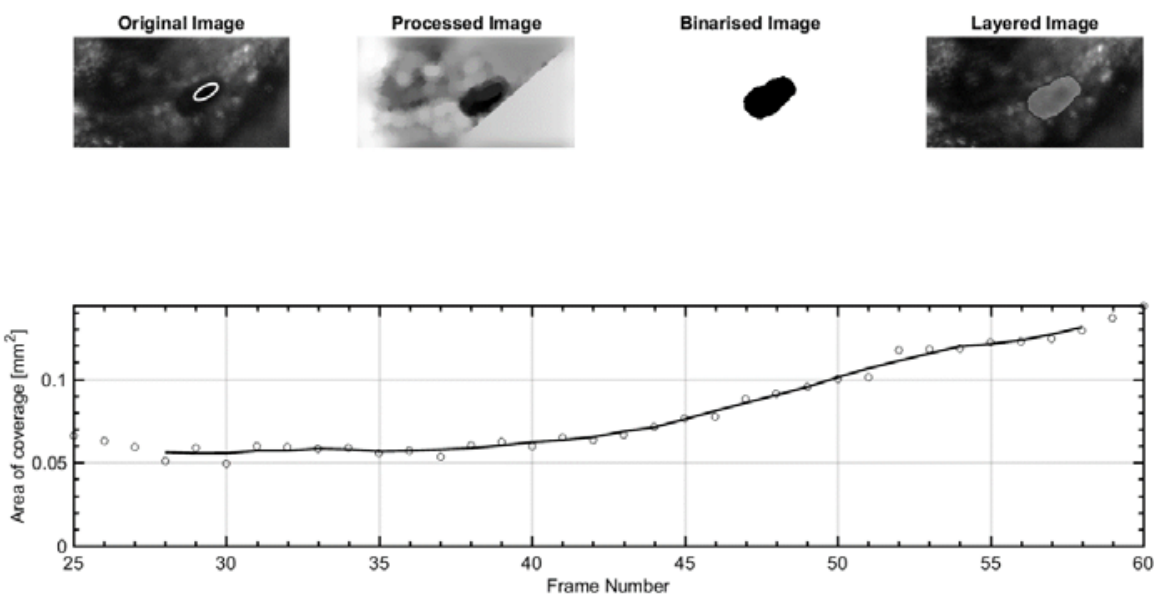

Figure 2. User interface showing the results of image analysis, with individual steps displayed so that the performance of the algorithm can be assessed in real time.

A mean value was extracted for data points between 3 and 3.5ms ASOI. This was able to yield an approximation for the initial quantitative coverage of the fluid, shown in Figure 3. The individual data points on that chart represent the mean values from all injections at matching injection pressures and injection timings in which coverage occurred. 


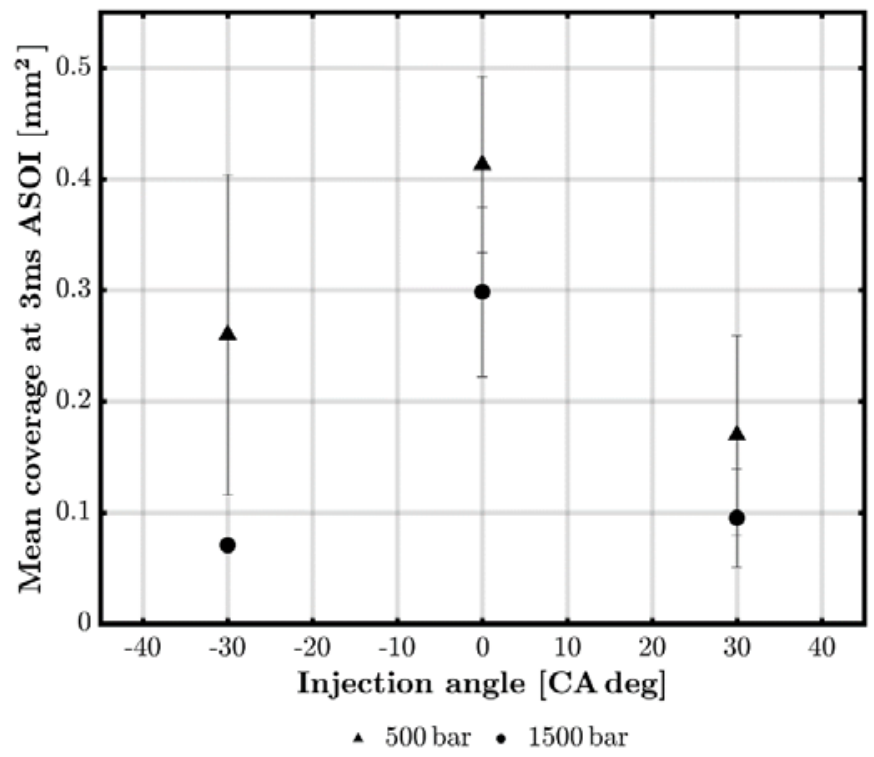

Figure 3. Mean initial coverage area measured $3 \mathrm{~ms}$ after the end of injection trigger. This shows that increasing injection pressure reduces the amount of initial fuel coverage once the spray has collapsed. Each data point represents the mean of 3 to

15 individual injections, and the error bars represent \pm 1 standard deviation. The orifice area has been subtracted.

\section{Results and discussion}

A key hypothesis that was tested in the present work is whether the presence of gas trapped inside the nozzle orifices could influence the wetting of the nozzle tip during the expansion stroke. As the in-cylinder pressure drops rapidly during expansion, gas trapped within the orifices should expand and displace liquid fuel onto the external surface of the nozzle tip. In order to test this hypothesis 3 injection timings were investigated:

1. During the compression stroke, when the gas pressure rise should inhibit the expansion of trapped gas

2. At top-dead-centre when the gas pressure is nearly constant

3. During the expansion stroke when the rapid gas pressure drop should promote the expansion of trapped gas.

The mean coverage area was found to depend heavily on the crank angle at which the injection pulse was sent. Injection at TDC is where the temperature and pressure are the greatest, higher pressure and temperatures relax contact angles and are conducive of more surface wetting. The lower in-cylinder pressures found when injecting away from TDC lead to greater fluid momentum at the instant when spray collapse occurs. This means there is less chance of splashback or ligament retraction towards the nozzle tip. It is interesting to note that the initial coverage areas measured when injecting during the compression (-30 CA) and expansion ( $+30 \mathrm{CA})$ strokes are almost identical. This confirms that the expansion of trapped gas does not significantly contribute to the initial wetting of this nozzle's surface.

The data recorded in Figure 3 shows a clear trend where increasing injection pressure leads to a reduction of the initial fuel coverage area. This can be explained by an increase of the fluid momentum at higher injection pressures, which in turn reduces the likelihood of ligament impingement or splashback onto the injector surface. The rate of liquid spreading was measured (Figure 4) by analysing the nozzle wetting shortly after the end of injection, using a linear regression where the gradient of the line represents the temporal evolution of the wetted surface area (Figure 5). Tthe same as those described for the initial coverage trend was observed for both injection timing and injection pressure. This suggests that the spreading rate highly correlates with the initial coverage area. This correlation indicates that the temporal increase in wetted surface area is due to the spreading of the initial liquid deposition, rather than the emergence of additional liquid from the orifice. This is further evidence that the expansion of orificetrapped gas bubbles is not the main contributor to liquid spreading across the near-nozzle surface after the end of injection. However, some exceptions were occasionally recorded, with the emergence of a bubble being observed and followed by an abrupt increase of the wetted area (Figure 5). Although such bubble expansions were not 
common, when they did occur, the coverage area could rapidly double; hence, the importance of this phenomenon may not be negligible.

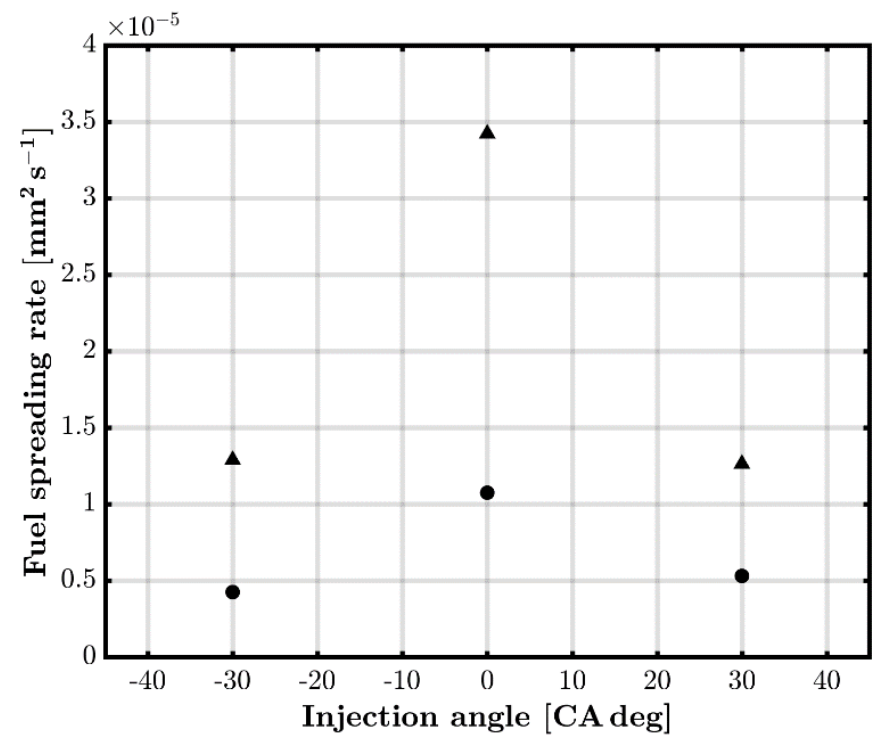

- 500 bar • 1500 bar

Figure 4. Illustration of how the rate of liquid spreading correlates with both crank angle and injection pressure. The rate of liquid spreading was calculated using linear regression analysis where the gradient of the line of best fit denotes the spreading rate in $\mathrm{mm}^{2} \mathrm{~s}^{-1}$ 


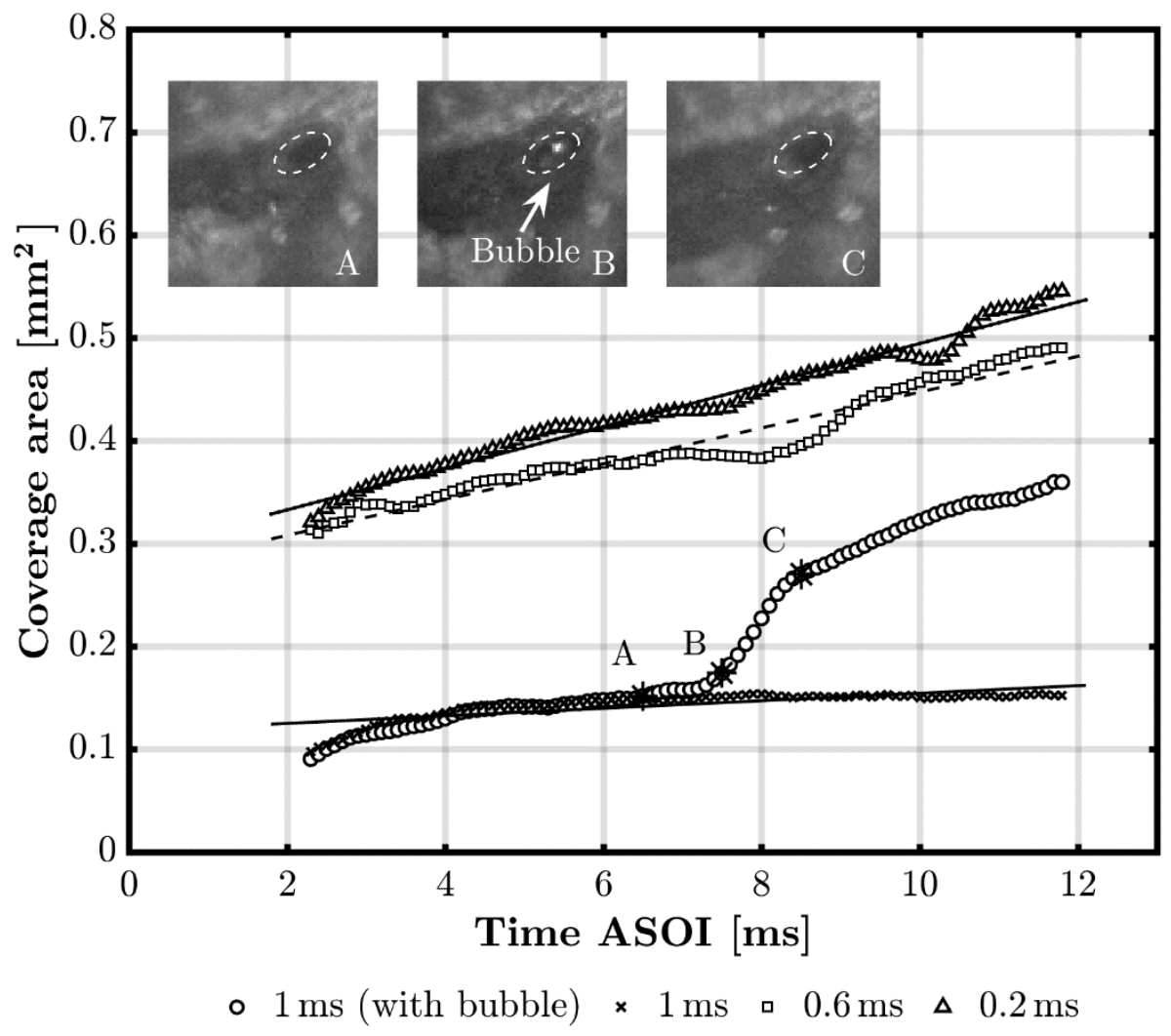

Figure 5. Temporal evolution of liquid coverage area as a function of time after start of injection trigger. In the majority of cases, the spreading rate observed was constant, as indicated by the linear trend lines. Occasionally, the emergence of a bubble was observed and followed by an abrupt increase of the detected coverage (white circles), however, this phenomena only occurred outside the image processing period. The dashed ellipses highlight the orifice location and the white arrow in frame B points to the distinctive scattering caused by the emergence of a liquid/gas interface within the orifice. Although such bubble expansions were not common, when they did occur, the coverage area rapidly increased.

\section{Conclusions}

In this study, a method for quantifying nozzle tip wetting after the end of injection was developed to gain a better understanding of the underlying processes and to study the influence of engine operating conditions. A high-speed camera with a long-distance microscope was used to visualise fluid behaviour at the microscopic scale during, and after, the end of injection. In order to measure the nozzle tip temperature a production injector was instrumented with a type $\mathrm{K}$ thermocouple near one of the orifices. Post-processing techniques were developed to track both the initial fuel coverage area on the nozzle surface, as well as the temporal evolution and spreading rate of surfacebound fluid.

Increasing the injection pressure from 500 bar to 1500 bar reduced the initial nozzle tip wetting. This is believed to be the result of increased momentum of the end of injection dribble, which reduces the likelihood of fluid contacting the surface of the near nozzle region, either by retraction of surface-bound ligaments or spray splashback. It was observed that the rate of spreading correlated with the initial area of fuel coverage measured after the end of injection, suggesting that the main mechanism for the wetting of a VCO nozzle is through the impingement of dribble onto the nozzle tip. 
For this nozzle the expansion of orifice-trapped gas bubbles was not found to be the main contributor to liquid spreading across the near-nozzle surface after the end of injection. However, on some occasions the emergence of a bubble was observed and followed by an abrupt increase of wetted surface area as liquid fuel inside the orifice was displaced onto the nozzle tip. When this occurred the coverage area could rapidly double, suggesting that this phenomenon may not be negligible.

\section{Acknowledgements}

This work was supported by BP Formulated Products Technology and the UK's Engineering and Physical Science Research Council [EPSRC grant EP/K020528/1 and EP/N509607/1].

\section{References}

1. H. Sandquist, I. Denbratt, F. Owrang, J. Olsson, Influence of fuel parameters on deposit formation and emissions in a direct injection stratified charge SI engine, in: SAE International Spring Fuels \& Lubricants Meeting, Orlando, Florida, USA, May 7-9, 2001. doi:10.4271/2001-01-2028.

2. R. E. Olsen, M. C. Ingham, G. M. Parsons, A fuel additive concentrate for removal of injector deposits in light-duty diesels, in: SAE International Fall Fuels \& Lubricants Meeting and Exhibition, Fairborn, Ohio, USA, October 20-23, 1984. doi:10.4271/841349.

3. R. Caprotti, W. J. Fowler, G. Lepperhoff, M. Houben, Diesel additive technology effects on injector hole erosion/corrosion, injector fouling and particulate traps, in: SAE International Fuels \& Lubricants Meeting \& Exposition, Philadelphia, Pennsylvania, USA, October 18-21, 1993. doi:10.4271/932739.

4. G. Lepperhoff, M. Houben, Mechanisms of deposit formation in internal combustion engines and heat exchangers, in: SAE International Congress \& Exposition, Detroit, Michigan, USA, October 17-20, 1993. doi:10.4271, 931032 .

5. A. Birgel, N. Ladommatos, P. Aleiferis, S. Zülch, N. Milovanovic, V. Lafon, A. Orlovic, P. Lacey, P. Richards, Deposit formation in the holes of diesel injector nozzles: A critical review, in: SAE Powertrains, Fuels \& Lubricants Meeting, Rosemont, Illinois, USA, October 6-9, 2008. doi:10.4271/2008-01-2383.

6. R. Caprotti, A. Breakspear, O. Graupner, T. Klaua, Detergency requirements of future diesel injection systems, in: SAE Powertrain \& Fluid Systems Conference \& Exhibition, San Antonio, Texas, USA, October 24-27, 2005. doi:10.4271/2005-01-3901.

7. B. Argueyrolles, S. Dehoux, P. Gastaldi, L. Grosjean, F. Levy, A. Michel, D. Passerel, Influence of injector nozzle design and cavitation on coking phenomenon, in: SAE International Fuels \& Lubricants Meeting, Kyoto, Japan, July 23-27, 2007. doi:10.4271/2007-01-1896.

8. J. Barker, C. Snape, D. Scurr, Information on the aromatic structure of internal diesel injector deposits from time of flight secondary ion mass spectrometry (ToF-SIMS), in: SAE 2014 World Congress \& Exhibition, Detroit, Michigan, USA, April 8-10, 2014. doi:10.4271/2014-01-1387.

9. J. Reid, S. Cook, J. Barker, Internal injector deposits from sodium sources, SAE International J. Fuels Lubricants 7 (2014) 436-44. doi:10.4271/2014-01-1388.

10. C. P. Koci, Y. Ra, R. Krieger, M. Andrie, D. E. Foster, R. M. Siewert, R. P. Durrett, Multiple-event fuel injection investigations in a highly-dilute diesel low temperature combustion regime, SAE International $\mathrm{J}$. Engines 2 (2009) 837-57. doi:10.4271/2009-01-0925.

11. C. Koci, A. Dempsey, J. Nudd, B. Knier, Understanding hydrocarbon emissions in heavy duty diesel engines combining experimental and computational methods, SAE International J. Engines 10 (2017). doi:10.4271, 2017-01-0703.

12. A. Swantek, D. Duke, N. Sovis, End of injection, mass expulsion behaviors in single hole diesel fuel injectors, in: Proceedings of ILASS Americas, Portland, Oregon, USA, September 18-21, 2014.

13. A. B. Swantek, A. L. Kastengren, D. Duke, F. Z. Tilocco, N. Sovis, C. F. Powell, A further examination of fuel dribble from single hole diesel nozzles, in: ILASS-Europe 26th Annual Conference, Bremen, Germany, September 8-10, 2014

14. S. Moon, W. Huang, Z. Li, J. Wang, End-of-injection fuel dribble of multi-hole diesel injector: Comprehensive investigation of phenomenon and discussion on control strategy, Applied Energy 179 (2016) 7-16. doi:10.1016/j.apenergy.2016.06.116.

15. W. E. Eagle, Cinema-stereo imaging of fuel dribble after the end of injection in an optical heavy-duty diesel engine, in: THIESEL, Valencia, Spain, September 9-12, 2014.

16. N. Papadopoulos, P. Aleiferis, Numerical modelling of the in-nozzle flow of a diesel injector with moving needle during and after the end of a full injection event, 12th International Conference on Engines \& Vehicles 8 (2015) 2285-302. doi:10.4271/2015-24-2472. 
17. M. Battistoni, A. L. Kastengren, C. F. Powell, S. Som, Fluid dynamics modeling of end-of-injection process, in: ILASS Americas 26th Annual Conference, Portland, Oregon, USA, May 5-8, 2014.

18. M. Battistoni, C. Poggiani, S. Som, Prediction of the nozzle flow and jet characteristics at start and end of injection: Transient behaviors, SAE International J. Engines 91 (2015) 84-97. doi:10.4271/2015-011850.

19. M. Battistoni, Q. Xue, S. Som, Large-eddy simulation (LES) of spray transients: Start and end of injection phenomena, Oil \& Gas Science Technology-Revue d'IFP Energies nouvelles 71 (2015) 1-4. doi:10.2516/ogst/2015024.

20. J. E. Turner, V. Stetsyuk, C. Crua, R. J. Pearson, M. R. Gold, The effect of operating conditions on postinjection fuel discharge in an optical engine, in: ICLASS 13th Triennial International Conference, Tainan, Taiwan, August 23-27, 2015.

21. D. R. Caudwell, J. P. M. Trusler, V. Vesovic, W. A. Wakeham, The viscosity and density of n-dodecane and n-octadecane at pressures up to $200 \mathrm{MPa}$ and temperatures up to $473 \mathrm{~K}$, International Journal of Thermophysics 25 (2004) 1339-52. doi:10.1007/s10765-004-5742-0. 